\title{
Electron localization functions and local measures of the covariance
}

\author{
PAUL W AYERS \\ Department of Chemistry, McMaster University, Hamilton, Ontario, Canada L8S 4M1 \\ e-mail: ayers@mcmaster.ca
}

\begin{abstract}
The electron localization measure proposed by Becke and Edgecombe is shown to be related to the covariance of the electron pair distribution. Just as with the electron localization function, the local covariance does not seem to be, in and of itself, a useful quantity for elucidating shell structure. A function of the local covariance, however, is useful for this purpose. A different function, based on the hyperbolic tangent, is proposed to elucidate the shell structure encapsulated by the local covariance; this function also seems to work better for the electron localization measure of Becke and Edgecombe. In addition, we propose a different measure for the electron localization that incorporates both the electron localization measure of Becke and Edgecombe and the Laplacian of the electron density; preliminary indications are that this measure is especially good at elucidating the shell structure in valence regions. Methods for evaluating electron localization functions directly from the electron density, without recourse to the Kohn-Sham orbitals, are discussed.
\end{abstract}

Keywords. Electron localization functions; local covariance; shell structure.

\section{Introduction}

The electron pair is perhaps the most useful concept in chemistry. Among the obvious related facts are "electron pairing" in covalent bonds, the octet and 18 -electron rules, and "lone pairs" of electrons. Many of these empirically useful rules are encapsulated in the Lewis "electron dot" structures and valence-shell electron pair repulsion (VSEPR) that pervade general chemistry texts and, indeed, the chemical research literature. ${ }^{1}$ The attempt to partition molecules into "electron pair regions" and thus obtain computationally the conceptually useful Lewis "picture" of molecules dates back to the pioneering studies of Daudel. ${ }^{2-5}$ One intuitively appealing criterion is that the number of electrons in an electron pair region, $\Omega$, should fluctuate relatively little, so that ${ }^{6-8}$

$$
\left\langle\Psi\left|\hat{N}_{\sigma}^{2}(\Omega)\right| \Psi\right\rangle-\left\langle\Psi\left|\hat{N}_{\sigma}(\Omega)\right| \Psi\right\rangle^{2}
$$

is small. Here we use $N_{\sigma}(\Omega)$ to represent the operator for the number of $\sigma$-spin electrons in the region $\Omega$,

$$
N(\Omega) \equiv \sum_{i=1}^{N}|\sigma(i)\rangle \chi_{\Omega}\left(r_{i}\right)\langle\sigma(i)|
$$

where $\chi_{\Omega}(r)$ is the characteristic function for the region $\Omega$,

$$
\chi_{\Omega}(\boldsymbol{r})= \begin{cases}1, & \boldsymbol{r} \in \Omega \\ 0, & \boldsymbol{r} \notin \Omega\end{cases}
$$

and $\langle\sigma(i)|$ is the projection operator for electron $i$ having spin $\sigma$. Bader and Stephens have noted that, despite its intuitive appeal, in many molecules the minimal fluctuation remains quite large and so this criterion sometimes fails to yield intuitive electron dot diagrams. ${ }^{9}$ The recent proposal by Savin and coworkers to define molecular regions be defined so that the probability of observing a chosen number of electrons in each region is maximized is related to the minimum fluctuation approach. ${ }^{10}$

An alternative construction of a Lewis-type pictures for molecules was proposed by Becke and Edgecombe, ${ }^{11-13}$ with an alternative subsequently proposed by Schmider and Becke. ${ }^{14,15}$ This approach is based on a consideration of the probability that an electron with spin $\sigma$ is a distance $s$ away from $\boldsymbol{r}$, given than there is another $\sigma$-spin electron at $\boldsymbol{r}$. Expressing the probability as

$$
P_{\sigma \sigma}(s \mid \boldsymbol{r}) \equiv \rho_{\sigma}(\boldsymbol{r})\left(\kappa\left[\rho_{\alpha}, \rho_{\beta}\right] s^{2}+\ldots\right)
$$

reveals that $\kappa[\rho]$ is a measure of how "localized" the Fermi hole is around the reference electron. In practice, because the core electrons are always the most localized, it is more interesting to consider where 
electrons are "especially localized". One does this by comparing to the result for a reference system, which is often taken to be the free electron gas with electron spin-density $\rho_{\sigma}(\boldsymbol{r}) .{ }^{11}$ This gives, as a measure of electron localization,

$$
\xi_{\sigma}(\boldsymbol{r}) \equiv \frac{\rho_{\sigma}(\boldsymbol{r}) \kappa\left[\rho_{\alpha}, \rho_{\beta}\right]}{(2 / 3)\left\{(3 / 10)\left(6 \pi^{2}\right)^{2 / 3} \rho_{\sigma}^{5 / 3}(\boldsymbol{r})\right\}} .
$$

The term in braces is just the Thomas-Fermi kinetic energy density. ${ }^{11,16-18}$ The form for $\kappa\left[\rho_{\alpha} \rho_{\beta}\right]$ differs depending on how one derives (4) ${ }^{19-22}$ The BeckeEdgecombe form is obtained by expressing the probability of observing a $\sigma$-spin electron at $\boldsymbol{r}^{\prime}$ when there is another $\sigma$-spin electron at $\boldsymbol{r}, P_{\sigma \sigma}\left(\boldsymbol{r}^{\prime} \mid \boldsymbol{r}\right)$, and then performing a spherical average with respect to $\left(\boldsymbol{r}^{\prime}-\boldsymbol{r}\right)$. If, in addition, one neglects the contribution to $P_{\sigma \sigma}\left(\boldsymbol{r}^{\prime} \mid \boldsymbol{r}\right)$ from the Coulomb hole, then

$$
P_{\sigma \sigma}\left(\boldsymbol{r}^{\prime} \mid \boldsymbol{r}\right) \equiv \frac{\gamma_{\sigma \sigma}\left(\boldsymbol{r}, \boldsymbol{r}^{\prime}\right) \gamma_{\sigma \sigma}\left(\boldsymbol{r}^{\prime}, \boldsymbol{r}\right)}{\rho(\boldsymbol{r})}
$$

where $\gamma_{\sigma \sigma}\left(\boldsymbol{r}, \boldsymbol{r}^{\prime}\right)$ is the Kohn-Sham density matrix for $\sigma$-spin electrons,

$$
\gamma_{\sigma \sigma}\left(\boldsymbol{r}, \boldsymbol{r}^{\prime}\right) \equiv \sum_{i=1}^{N_{\sigma}} \psi_{i \sigma}^{*}\left(\boldsymbol{r}^{\prime}\right) \psi_{i \sigma}(\boldsymbol{r})
$$

and $\left(\psi_{i \sigma}\right)$ are the $\sigma$-spin orbitals solving the KohnSham equations. This gives ${ }^{11}$

$$
\kappa_{\sigma}^{B E}\left[\rho_{\alpha}, \rho_{\beta}\right] \equiv \frac{2}{3} \frac{\tau_{\sigma}(\boldsymbol{r})-\tau_{\sigma}^{W}(\boldsymbol{r})}{\rho_{\sigma}(\boldsymbol{r})},
$$

where the positive-definite kinetic energy density is defined by

$$
\tau_{\sigma}(\boldsymbol{r}) \equiv \sum_{i=1}^{N_{\sigma}} \frac{\nabla \psi_{i \sigma}^{*}(\boldsymbol{r}) \cdot \nabla \psi_{i \sigma}^{*}(\boldsymbol{r})}{2},
$$

and the "Weisacker kinetic energy density" is defined by

$$
\tau_{\sigma}^{W}(\boldsymbol{r}) \equiv \frac{\nabla \rho_{\sigma}(\boldsymbol{r}) \cdot \nabla \rho_{\sigma}(\boldsymbol{r})}{8 \rho_{\sigma}(\boldsymbol{r})}=\frac{\nabla \sqrt{\rho_{\sigma}(\boldsymbol{r})} \cdot \nabla \sqrt{\rho_{\sigma}(\boldsymbol{r})}}{2} .
$$

Equations (6)-(10) represent the results in the "exchange-only" approximation, are and exact for sys- tems of non-interacting electrons, where the only structure to the electron-electron distribution function originates in antisymmetry, as dictated by the Pauli principle.

The Schmider-Becke form is obtained by spherically averaging $\gamma_{\sigma \sigma}\left(\boldsymbol{r}, \boldsymbol{r}^{\prime}\right)$ about $\left(\boldsymbol{r}^{\prime}-\boldsymbol{r}\right)$ and then substituting into the analogue of $(6)$, yielding ${ }^{23}$

$$
\kappa_{\sigma}^{S B}\left[\rho_{\alpha}, \rho_{\beta}\right] \equiv \frac{2}{3}\left[\tau_{\sigma}(\boldsymbol{r}) / \rho_{\sigma}(\boldsymbol{r})\right] .
$$

Equation (11) has also been rationalized using local thermodynamics. ${ }^{24,25}$ Indeed, the Becke-Edgecombe and Schmider-Becke electron localization functions can each be derived from many different approaches, owing to the fact that functions related to the Fermi hole are similar to $P_{\sigma \sigma}(s \mid \boldsymbol{r})$ through order $s^{2}$, typically differing only in higher orders. For example, the following approaches yield the same fundamental measures of electron localization (through second order in $s$ ):

(a) Conventional ELF: Given that a $\sigma$-spin electron is at $\boldsymbol{r}$, what is the probability another electron with the same spin is $s$ units from $r$ ?

(b) Centre of Mass ELF: Given that the center of mass of two $\sigma$-spin electrons is at $\boldsymbol{r}$, what is the probability the electrons are $s$ units apart?

(c) Pair regions ELF: What is the probability that two $\sigma$-spin electrons are within $s$ units of $\boldsymbol{r} ?^{26} \mathrm{~A}$ variant of this approach, where the probability of observing a pair of $\sigma$-spin electrons in a small cubic volume around the point, $\boldsymbol{r}$, was considered by Kohout. ${ }^{27}$

The last approach, based on the probability of observing electron pairs in small regions of space, is especially appealing since a divisor proportional to $\rho_{\sigma}^{5 / 3}(\boldsymbol{r})$ naturally arises. This obviates the need to appeal to the free electron gas.

The Becke-Edgecombe approach has the advantage of addressing the exchange-correlation hole directly and, in the author's experience, tends to give a more distinct separation of electron-pair regions. For this reason, the present paper is focused on the Becke-Edgecombe form.

Here we seek to answer several questions related to the topic of electron localization measures. First of all, how should we divide a molecule into electron pair regions? We address this question in $\S \S 2.1$ and 2.2. Pertaining to (5), the Thomas-Fermi reference seems arbitrary. Does this reference have some 
physical significance and, if not, would other reference kinetic energy densities work equally well? In $\S \S 2.3$ and 2.4 , we show that, quite apart from the Thomas-Fermi model, a term proportional to $\rho^{5 / 3}(\boldsymbol{r})$ belongs in the denominator of (5). Finally, evaluating the kinetic energy density requires knowledge of the Kohn-Sham orbitals. ${ }^{28}$ Such orbitals, however, are not immediately accessible from standard techniques for calculating correlated wave functions, and require a subsequent calculation to discern the Kohn-Sham orbitals from the correlated density. ${ }^{29-32}$ Electron localization measures can be computed directly from the correlated electron density, without recourse to the Kohn-Sham orbitals, ${ }^{33-35}$ and in $\$ 2.5$ we discuss this approach and offer a small refinement. Throughout these sections, we shall use the neon atom as a representative example. In $\$ 2.6$, we extend our analysis to the argon, krypton, and xenon atoms.

Readers who are primarily interested in the final results, rather than their theoretical motivations, will find key results derived in $\S \S 2.3$ through 2.5 , applied in $\S 2.6$, and summarized in $\S 3$.

\section{Theoretical results}

\subsection{The norm of the covariance matrix as a measure of electron localization}

We wish to derive a new electron localization measure that, though directly related to the correlation between and fluctuation of the electron distribution, is different from existing approaches. We start by listing several key properties a "good" partitioning of a molecule into electron-pair regions should possess:

(i) The molecule should be divided into $N_{\sigma}$ regions, $\left\{\Omega_{i}\right\}_{i=1}^{N_{\sigma}}$ and each region should contain, on average, one $\sigma$-spin electron.

(ii) The regions should be "weakly coupled". A simple and computationally useful criterion is that the covariance of the number of $\sigma$-spin electrons in regions $\Omega_{i}$ and $\Omega_{j}$ should be small

$$
\begin{aligned}
\sigma_{i j}^{2} \equiv & \left\langle\Psi\left|\hat{N}_{\sigma}\left(\Omega_{i}\right) \hat{N}_{\sigma}\left(\Omega_{j}\right)\right| \Psi\right\rangle \\
& -\left\langle\Psi\left|\hat{N}_{\sigma}\left(\Omega_{i}\right)\right| \Psi\right\rangle\left\langle\Psi\left|\hat{N}_{\sigma}\left(\Omega_{j}\right)\right| \Psi\right\rangle \approx 0
\end{aligned}
$$

This reflects the Lewis picture, whereby electrons within a pair are highly correlated, but electrons in different pairs are only weakly correlated. For instance, if $\sigma_{i j}^{2}=0$, then the number of electrons in $\Omega_{i}$ is, to first order, independent of the number of electrons in $\Omega_{j}$. This is consistent with the Lewis picture, wherein "electron pairs" interact only weakly with one another.

(iii) The fluctuation in the electron number, as given by (1), should be minimal. Thus, among partitionings which satisfy constraints (i) and (ii), we are interested in those with the smallest possible values of

$$
\sigma_{i i}^{2} \equiv\left\langle\Psi\left|\hat{N}_{\sigma}^{2}\left(\Omega_{i}\right)\right| \Psi\right\rangle-\left\langle\Psi\left|\hat{N}_{\sigma}\left(\Omega_{i}\right)\right| \Psi\right\rangle^{2} .
$$

Several points should be mentioned. First of all, we will typically have ${ }^{+}$

$$
\sigma_{i j}^{2}<0, \quad i \neq j
$$

It should also be noted that

$$
\begin{aligned}
\sum_{j} \sigma_{i j}^{2} \equiv \sum_{j}\left[\int_{\Omega_{j}} \int_{\Omega_{i}} \rho_{\sigma}(\boldsymbol{r}) \rho_{\sigma}\left(\boldsymbol{r}^{\prime}\right) g_{\sigma \sigma}\left(\boldsymbol{r}, \boldsymbol{r}^{\prime}\right) \mathrm{d} \boldsymbol{r} \mathrm{d} \boldsymbol{r}^{\prime}\right. \\
\left.\quad-\int_{\Omega_{j}} \rho_{\sigma}(\boldsymbol{r}) \mathrm{d} \boldsymbol{r} \int_{\Omega_{i}} \rho_{\sigma}(\boldsymbol{r}) \mathrm{d} \boldsymbol{r}\right]+\int_{\Omega_{i}} \rho_{\sigma}(\boldsymbol{r}) \mathrm{d} \boldsymbol{r} \\
=\left(N_{\sigma}-1\right) \int_{\Omega_{i}} \rho_{\sigma}(\boldsymbol{r}) \mathrm{d} \boldsymbol{r}-N_{\sigma} \int_{\Omega_{i}} \rho_{\sigma}(\boldsymbol{r}) \mathrm{d} \boldsymbol{r} \\
+\int_{\Omega_{i}} \rho_{\sigma}(\boldsymbol{r}) \mathrm{d} \boldsymbol{r}=0,
\end{aligned}
$$

where $g_{\sigma \sigma}\left(\boldsymbol{r}, \boldsymbol{r}^{\prime}\right)=1+1 h_{\sigma \sigma}\left(\boldsymbol{r}, \boldsymbol{r}^{\prime}\right)$ is the pair correlation function and

$$
\begin{aligned}
\rho_{2}^{\sigma \sigma}\left(\boldsymbol{r}, \boldsymbol{r}^{\prime}\right) \equiv & \rho_{\sigma}(\boldsymbol{r}) \rho_{\sigma}\left(\boldsymbol{r}^{\prime}\right) g_{\sigma \sigma}\left(\boldsymbol{r}, \boldsymbol{r}^{\prime}\right) \\
\equiv & \rho_{\sigma}(\boldsymbol{r}) \rho_{\sigma}\left(\boldsymbol{r}^{\prime}\right)\left(1+h_{\sigma \sigma}\left(\boldsymbol{r}, \boldsymbol{r}^{\prime}\right)\right) \\
\equiv\left\langle\Psi\left|\sum_{j \neq i}^{N} \sum_{i=1}^{N}\right| \sigma(i) \sigma(j)\right\rangle \delta\left(\boldsymbol{r}_{i}-\boldsymbol{r}\right) & \\
& \times \delta\left(\boldsymbol{r}_{j}-\boldsymbol{r}^{\prime}\right)\langle\sigma(i) \sigma(j)|\Psi|\rangle,
\end{aligned}
$$

\footnotetext{
${ }^{+}$Observing an electron in the region $\Omega_{i}$ tends to decrease the probability of observing an electron in the region $\Omega_{j}$, mostly because there are only $N-1$ electrons "left" to be in this region. More precisely, due to the Pauli exclusion principle, the Fermi hole indicates that the hole correlation function is predominately negative and, if the hole correlation function were strictly negative, (14) would always be true.
} 
is the pair distribution function or "pair density." Equations (14) and (15), together, imply that

$$
\sigma_{i i}^{2}>0
$$

A key implication of (15) is that the minimum covariance problem (criterion (ii)) and the minimum electron-number fluctuation problem (criterion (iii)) are one and the same.

To derive (15), use the normalization of the pair correlation function,

$$
\int \rho_{\sigma}(\boldsymbol{r}) g_{\sigma \sigma}\left(\boldsymbol{r}, \boldsymbol{r}^{\prime}\right) \mathrm{d} \boldsymbol{r}=N_{\sigma}-1,
$$

along with the result for the variance,

$$
\begin{aligned}
& \sigma_{i i}^{2} \equiv\left\langle\Psi\left|\hat{N}_{\sigma}^{2}\left(\Omega_{i}\right)\right| \Psi\right\rangle-\left\langle\Psi\left|\hat{N}_{\sigma}\left(\Omega_{i}\right)\right| \Psi\right\rangle^{2} \\
& =\left\langle\Psi\left|\sum_{l=1}^{N} \sum_{k=1}^{N}\right| \sigma(k) \boldsymbol{\sigma}(l)\right\rangle \chi_{\Omega_{i}}\left(\boldsymbol{r}_{k}\right) \chi_{\Omega_{i}}\left(\boldsymbol{r}_{l}\right)\langle\boldsymbol{\sigma}(k) \boldsymbol{\sigma}(l) \mid \Psi\rangle \\
& \left.-\left\langle\Psi\left|\sum_{k=1}^{N}\right| \sigma(k)\right\rangle \chi_{\Omega_{i}}\left(\boldsymbol{r}_{k}\right)\langle\sigma(k) \mid \Psi\rangle\right\rangle^{2} \\
& =\left\langle\Psi\left|\sum_{l \neq k}^{N} \sum_{k=1}^{N}\right| \sigma(k) \sigma(l)\right\rangle \chi_{\Omega_{i}}\left(\boldsymbol{r}_{k}\right) \chi_{\Omega_{i}}\left(\boldsymbol{r}_{l}\right)\langle\boldsymbol{\sigma}(k) \sigma(l)|| \Psi\rangle \\
& +\left\langle\Psi\left|\sum_{k=1}^{N}\right| \sigma(k) \sigma(k)\right\rangle \chi_{\Omega_{i}}\left(\boldsymbol{r}_{k}\right) \chi_{\Omega_{i}}\left(\boldsymbol{r}_{k}\right)\langle\sigma(k) \boldsymbol{\sigma}(k)|| \Psi\rangle \\
& -\left(\int_{\Omega_{i}} \rho_{\sigma}(\boldsymbol{r}) \mathrm{d} \boldsymbol{r}\right)^{2} \\
& =\int_{\Omega_{i}} \int_{\Omega_{i}} \rho_{\sigma}(\boldsymbol{r}) \rho_{\sigma}\left(\boldsymbol{r}^{\prime}\right) g_{\sigma \sigma}\left(\boldsymbol{r}, \boldsymbol{r}^{\prime}\right) \mathrm{d} \boldsymbol{r} \mathrm{d} \boldsymbol{r}^{\prime} \\
& +\left\langle\Psi\left|\sum_{k=1}^{N}\right| \sigma(k)\right\rangle \chi_{\Omega_{i}}\left(\boldsymbol{r}_{k}\right)\langle\sigma(k) \mid \Psi\rangle \\
& -\left(\int_{\Omega_{i}} \rho_{\sigma}(\boldsymbol{r}) \mathrm{d} \boldsymbol{r}\right)^{2} \\
& =\int_{\Omega_{i}} \int_{\Omega_{i}} \rho_{\sigma}(\boldsymbol{r}) \rho_{\sigma}\left(\boldsymbol{r}^{\prime}\right) g_{\sigma \sigma}\left(\boldsymbol{r}, \boldsymbol{r}^{\prime}\right) \mathrm{d} \boldsymbol{r} \mathrm{d} \boldsymbol{r}^{\prime} \\
& +\int_{\Omega_{i}} \rho_{\sigma}(\boldsymbol{r}) \mathrm{d} \boldsymbol{r}-\left(\int_{\Omega_{i}} \rho_{\sigma}(\boldsymbol{r}) \mathrm{d} \boldsymbol{r}\right)^{2} .
\end{aligned}
$$

For a Slater determinate wave function (where $\left.h_{\sigma \sigma}\left(\boldsymbol{r}, \boldsymbol{r}^{\prime}\right) \leq 0\right)$, a necessary and sufficient condition for $\sigma_{i j}^{2}=\sigma_{i i}^{2}=0$ is that the hole correlation function be strictly localized in the regions $\left\{\Omega_{i}\right\}$, so that the hole correlation function is always zero when $\boldsymbol{r}$ and $r^{\prime}$ are in different regions:

$$
h_{\sigma \sigma}\left(\boldsymbol{r}, \boldsymbol{r}^{\prime}\right)=0, \quad \boldsymbol{r} \in \Omega_{i} \text { and } \boldsymbol{r}^{\prime} \notin \Omega_{i} .
$$

Equation (20) is only true if electrons in different regions are totally uncorrelated.

It follows from the previous considerations that a "good partitioning" of a molecule into electron-pair regions can be obtained by choosing regions, $\left\{\Omega_{i}\right\}$, such that the norm of the covariance matrix, $\sigma_{i j}^{2}$ is small. That is, the optimal regions minimize $\left\|\sigma_{i j}^{2}\right\|$. The choice of norm one adopts depends on how one wishes to measure the "non-locality" of a given covariance matrix. Perhaps the simplest choice is the Frobenius norm, which is simply the square root of the sums of the squares of the elements of the covariance matrix,

$$
\left\|\sigma_{i j}^{2}\right\|_{F} \equiv\left(\sum_{i, j}\left(\sigma_{i j}^{2}\right)^{2}\right)^{1 / 2},
$$

subject to the constraint (cf. (i)) that

$$
\int_{\Omega_{i}} \rho_{\sigma}(\boldsymbol{r}) \mathrm{d} \boldsymbol{r}=1
$$

From (15), we see that

$$
\begin{aligned}
\left\|\sigma_{i j}^{2}\right\|_{F}^{2} & =\sum_{i} \sum_{j \neq i}\left(\sigma_{i j}^{2}\right)^{2}+\sum_{i=1}\left(\sigma_{i i}^{2}\right)^{2} \\
& =2 \sum_{i} \sum_{j \neq i}\left(\sigma_{i j}^{2}\right)^{2}=2 \sum_{i=1}\left(\sigma_{i i}^{2}\right)^{2}
\end{aligned}
$$

and $\left\|\sigma_{i j}^{2}\right\|_{F}$ can be minimized by considering only the diagonal or the off-diagonal elements of the covariance matrix.

We postulate, based on the preceding theoretical considerations, that the regions that minimize (21) will give useful electron pair "loges". However, performing computational tests of these ideas is difficult (mostly because the characteristic functions, (3), to be optimized are discontinuous) and will not be pursued here.

\subsection{Pointwise measures of covariance}

Insofar as the purpose of the electron localization function is to provide qualitative information about 
electron pairing, undertaking time-consuming calculations (as minimizing the norm of the covariance matrix, (21), seems to entail), seems inappropriate. In the spirit of the above analysis, however, we can derive various local measures of the fluctuation and covariance, and these measures seem to be much more tractable computationally than the electron localization techniques discussed in the previous section. In this section, two convenient measures of local covariance are proposed.

Towards this goal, note that the number of $\sigma$-spin electrons at $\boldsymbol{r}_{i}$ given that there is an $\sigma$-spin electrons electron at $\boldsymbol{r}_{j}$ is $\left(\rho_{2}^{\sigma \sigma}\left(\boldsymbol{r}_{i}, \boldsymbol{r}_{j}\right) / \rho_{\sigma}\left(\boldsymbol{r}_{j}\right)\right) \mathrm{d} \boldsymbol{r}_{i}$, while the average number of $\sigma$-spin electrons at $\boldsymbol{r}_{i}$ is $\rho_{\sigma}\left(\boldsymbol{r}_{i}\right) \mathrm{d} \boldsymbol{r}_{i}$. Given a $\sigma$-spin electron at $\boldsymbol{r}_{j}$, the amount by which the number of $\sigma$-spin electrons at $\boldsymbol{r}_{i}$ differs from its average value is

$$
N_{\sigma}\left(\boldsymbol{r}_{i}\right)-\overline{N_{\sigma}\left(\boldsymbol{r}_{i}\right)}=\rho_{2}^{\sigma \sigma}\left(\boldsymbol{r}_{i}, \boldsymbol{r}_{j}\right) / \rho_{\sigma}\left(\boldsymbol{r}_{j}\right)-\rho_{\sigma}\left(\boldsymbol{r}_{i}\right)
$$

and the total "fluctuation" in the electron number at $\boldsymbol{r}_{i}$ per electron,

$$
\zeta_{\sigma}^{h}\left[\rho ; \boldsymbol{r}_{i}\right] \equiv\left[N_{\sigma}\left(\boldsymbol{r}_{i}\right)-\overline{N_{\sigma}\left(\boldsymbol{r}_{i}\right)}\right]^{2} / \overline{N_{\sigma}\left(\boldsymbol{r}_{i}\right)}
$$

is then obtained by integrating over all possible values of $\boldsymbol{r}_{j}$ :

$$
\begin{aligned}
& \zeta_{\sigma}^{h}\left[\rho ; \boldsymbol{r}_{i}\right] \\
& =\left\{\left[\int\left(\frac{\rho_{2}^{\sigma \sigma}\left(\boldsymbol{r}_{i}, \boldsymbol{r}_{j}\right)}{\rho_{\sigma}\left(\boldsymbol{r}_{j}\right)} \rho_{\sigma}\left(\boldsymbol{r}_{i}\right)\right)^{2} \mathrm{~d} \boldsymbol{r}_{j}\right] \mathrm{d} \boldsymbol{r}_{i}\right\} / \rho_{\sigma}\left(\boldsymbol{r}_{i}\right) \mathrm{d} \boldsymbol{r}_{i} \\
& =\rho_{\sigma}\left(\boldsymbol{r}_{i}\right) \int\left(h_{\sigma \sigma}\left(\boldsymbol{r}_{i}, \boldsymbol{r}_{j}\right)\right)^{2} \mathrm{~d} \boldsymbol{r}_{j} .
\end{aligned}
$$

Because the exact hole correlation function is bounded and decays asymptotically as $\left|\boldsymbol{r}_{i}-\boldsymbol{r}_{j}\right|^{-5}, 36$ the integral in (26) exists. However, for approximate models, the integral in (26) might be infinite. In practice, I have observed that as the reference point, $\boldsymbol{r}_{i}$, moves away from an atom's nucleus, it becomes practically impossible to numerically evaluate the integral in (26). This is probably because when the electron density at $\boldsymbol{r}_{i}$ is small, the normalization constraint on the exchange hole requires $h\left(\boldsymbol{r}_{i}, \boldsymbol{r}_{j}\right)$ to be very diffuse, complicating the evaluation of $\zeta_{\sigma}^{h}\left[\rho ; \boldsymbol{r}_{i}\right]$.

The interpretation of $\zeta_{\sigma}^{h}\left(\boldsymbol{r}_{i}\right)$ is simple: $\zeta_{\sigma}^{h}\left(\boldsymbol{r}_{i}\right)$ measures the relative amount by which the other electrons in the system cause the electron density at $\boldsymbol{r}_{i}$ to differ from its average value. The decision to use the average per electron change in electron population at $\boldsymbol{r}_{i}$ due to the presence of an electron at $\boldsymbol{r}_{j}$ rather than the total change - that is, the decision to divide by $\rho_{\sigma}\left(\boldsymbol{r}_{i}\right) \mathrm{d} \boldsymbol{r}_{i}$ in (26) - is motivated by the recognition that where the electron density is very small (as in the asymptotic regions) the total fluctuation observed will be very small, even though the relative fluctuation can be quite large. Consequently, it seems prudent to use the fluctuation per electron of the conditional probability, rather than its absolute value. Computational "experiments" reveal this to be a reasonable decision.

The preceding analysis focused on minimizing the fluctuation in the conditional distribution function, and was motivated by the consideration of this function in the work of Becke and Edgecombe. To show the relationship between minimizing the Frobenius norm of the covariance matrix, (21), and (26), consider a set of $P$ distinct regions $\left\{\Omega_{i}\right\}_{i=1}^{P}$ which "cover" the molecule (or at least all the molecular regions that containing significant electron density). We have that

$$
\left\langle N_{\sigma}\left(\Omega_{i}\right) N_{\sigma}\left(\Omega_{j}\right)\right\rangle=\iint_{\substack{r_{i} \in \Omega_{i} \\ r_{j} \in \Omega_{j}}} \rho_{2}^{\sigma \sigma}\left(\boldsymbol{r}_{i}, \boldsymbol{r}_{j}\right) \mathrm{d} \boldsymbol{r}_{i} \mathrm{~d} \boldsymbol{r}_{j}
$$

and

$$
\left\langle N_{\sigma}\left(\Omega_{i}\right)\right\rangle\left\langle N_{\sigma}\left(\Omega_{j}\right)\right\rangle=\iint_{\substack{r_{i} \in \Omega_{i} \\ r_{j} \in \Omega_{j}}} \rho_{\sigma}\left(\boldsymbol{r}_{i}\right) \rho_{\sigma}\left(\boldsymbol{r}_{j}\right) \mathrm{d} \boldsymbol{r}_{i} \mathrm{~d} \boldsymbol{r}_{j} .
$$

As the regions $\left\{\Omega_{i}\right\}_{i=1}^{P}$ become smaller and smaller, we can approximate the integration of a function over $\Omega_{i}$ by a value the function assumes on the region multiplied by the volume of the region. In the limit of infinitesimal regions, we have

$$
\sigma_{i j}^{2}=\left(\rho_{2}^{\sigma \sigma}\left(\boldsymbol{r}_{i}, \boldsymbol{r}_{j}\right)-\rho_{\sigma}\left(\boldsymbol{r}_{i}\right) \rho_{\sigma}\left(\boldsymbol{r}_{j}\right)\right) \mathrm{d} \boldsymbol{r}_{i} \mathrm{~d} \boldsymbol{r}_{j},
$$

where $\mathrm{d} \boldsymbol{r}_{i}$ and $\mathrm{d} \boldsymbol{r}_{j}$ are the differential volumes of the regions and $\boldsymbol{r}_{i} \in \Omega_{i}$ and $\boldsymbol{r}_{j} \in \Omega_{j}$. Equation (26) is seen to be a differential analogue of the formula,

$$
\zeta_{i}^{h} \equiv\left\{\sum_{j \neq i}^{P}\left(\frac{\sigma_{i j}^{2}}{N_{j}}\right)^{2}\right\} / N_{i},
$$

where $N_{j}$ denotes the number of electrons in $\Omega_{i}$. Using (23), it is not difficult to show that $\zeta_{i}^{h}=0$ if and only if $\left\|\sigma_{i j}^{2}\right\|_{F}=0$. 
We now propose a different measure for electron localization: one which is closer to the spirit of the results in \$2.1. To do this, we form the correlation matrix,

$$
R_{i j} \equiv \sigma_{i j}^{2} /\left(\sqrt{\sigma_{i i}^{2}} \sqrt{\sigma_{j j}^{2}}\right)
$$

The number of $\sigma$-spin electrons in region $\Omega_{i}, N_{\sigma}\left(\Omega_{i}\right)$, is independent of the number of electrons in the region, $\Omega_{i}$, if $R_{i j} \equiv 0$. Consequently, minimizing the fluctuation in the number of electrons in region $\Omega_{i}$ entails minimizing

$$
\sum_{j \neq i}^{P} R_{i j}^{2}
$$

and the total overall fluctuation can be minimized by minimizing $\|\boldsymbol{R}-\boldsymbol{I}\|_{F}$, where $\boldsymbol{I}$ is the identity matrix. Considering the correlation matrix instead of the covariance matrix has a major advantage when we consider the limit where the regions, $\left\{\Omega_{i}\right\}_{i=1}^{P}$, become volume elements for Riemann integration. Unlike the covariance matrix, whereby the fluctuation per electron goes to zero as the volume if the regions approach zero,

$$
\begin{aligned}
& \lim _{V\left(\Omega_{i}\right) \rightarrow 0}\left(\sum_{j \neq i}^{P}\left(\sigma_{i j}^{2}\right)^{2}\right) / N_{\sigma}\left(\Omega_{i}\right) \\
& \rightarrow \sum_{j \neq i}^{P} \frac{\left[\left(\rho_{2}^{\sigma \sigma}\left(\boldsymbol{r}_{i}, \boldsymbol{r}_{j}\right)-\rho_{\sigma}\left(\boldsymbol{r}_{i}\right) \rho_{\sigma}\left(\boldsymbol{r}_{j}\right)\right) \mathrm{d} \boldsymbol{r}_{i} \mathrm{~d} \boldsymbol{r}_{j}\right]^{2}}{\rho_{\sigma}\left(\boldsymbol{r}_{i}\right) \mathrm{d} \boldsymbol{r}_{j}} \\
& \rightarrow 0,
\end{aligned}
$$

the zero-volume limit of the per-electron correlation at $\boldsymbol{x}$ gives the useful result

$$
\begin{gathered}
\lim _{V\left(\Omega_{i}\right) \rightarrow 0} \sum_{j \neq i}^{P} R_{i j}^{2} / N\left(\Omega_{i}\right) \\
=\frac{1}{\rho_{\sigma}\left(\boldsymbol{r}_{i}\right) \mathrm{d} \boldsymbol{r}_{i}} \\
\sum_{j \neq i} \frac{\left[\left(\rho_{2}^{\sigma \sigma}\left(\boldsymbol{r}_{i}, \boldsymbol{r}_{j}\right)-\rho_{\sigma}\left(\boldsymbol{r}_{i}\right) \rho_{\sigma}\left(\boldsymbol{r}_{j}\right)\right) \mathrm{d} \boldsymbol{r}_{i} \mathrm{~d} \boldsymbol{r}_{j}\right]^{2}}{\left(\rho_{\sigma}\left(\boldsymbol{r}_{i}\right) \mathrm{d} \boldsymbol{r}_{i}-\left(\rho_{\sigma}\left(\boldsymbol{r}_{i}\right) \mathrm{d} \boldsymbol{r}_{i}\right)^{2}\right)\left(\rho_{\sigma}\left(\boldsymbol{r}_{j}\right) \mathrm{d} \boldsymbol{r}_{j}-\left(\rho_{\sigma}\left(\boldsymbol{r}_{j}\right) \mathrm{d} \boldsymbol{r}_{j}\right)^{2}\right)} \\
=\frac{1}{\rho_{\sigma}\left(\boldsymbol{r}_{i}\right) \mathrm{d} \boldsymbol{r}_{i}} \\
\quad \times \sum_{j \neq i} \frac{\left[\rho_{\sigma}\left(\boldsymbol{r}_{i}\right) \rho_{\sigma}\left(\boldsymbol{r}_{j}\right) h_{\sigma \sigma}\left(\boldsymbol{r}_{i}, \boldsymbol{r}_{j}\right) \mathrm{d} \boldsymbol{r}_{i} \mathrm{~d} \boldsymbol{r}_{j}\right]^{2}}{\rho_{\sigma}\left(\boldsymbol{r}_{i}\right) \rho_{\sigma}\left(\boldsymbol{r}_{j}\right) \mathrm{d} \boldsymbol{r}_{i} \mathrm{~d} \boldsymbol{r}_{j}} \\
=\int \rho_{\sigma}\left(\boldsymbol{r}_{j}\right) h_{\sigma \sigma}^{2}\left(\boldsymbol{r}_{i}, \boldsymbol{r}_{j}\right) \mathrm{d} \boldsymbol{r}_{j},
\end{gathered}
$$

where, as in (28), $\boldsymbol{r}_{i} \in \Omega_{i}$ and $\boldsymbol{r}_{j} \in \Omega_{j}$. In evaluating (34), we used the fact that (cf. (19)),

$$
\begin{aligned}
\underbrace{\lim }_{\left(\Omega_{i}\right) \rightarrow 0} \sigma_{i i}^{2} \equiv & \rho_{\sigma}\left(\boldsymbol{r}_{i}\right) \rho_{\sigma}\left(\boldsymbol{r}_{i}\right) g_{\sigma \sigma}\left(\boldsymbol{r}_{i}, \boldsymbol{r}_{i}\right) \mathrm{d} \boldsymbol{r}_{i} \mathrm{~d} \boldsymbol{r}_{i} \\
& +\rho_{\sigma}\left(\boldsymbol{r}_{i}\right) \mathrm{d} \boldsymbol{r}_{i}-\left(\rho_{\sigma}\left(\boldsymbol{r}_{i}\right) \mathrm{d} \boldsymbol{r}_{i}\right)^{2} \\
= & \rho_{\sigma}\left(\boldsymbol{r}_{i}\right) \mathrm{d} \boldsymbol{r}_{i}-\left(\rho_{\sigma}\left(\boldsymbol{r}_{i}\right) \mathrm{d} \boldsymbol{r}_{i}\right)^{2},
\end{aligned}
$$

because $g_{\sigma \sigma}\left(\boldsymbol{r}_{i}, \boldsymbol{r}_{i}\right)=0$.

By this argument, the index

$$
\zeta_{\sigma}^{R}\left(\boldsymbol{r}_{i}\right) \equiv \int \rho_{\sigma}\left(\boldsymbol{r}_{j}\right) h_{\sigma \sigma}^{2}\left(\boldsymbol{r}_{i}, \boldsymbol{r}_{j}\right) \mathrm{d} \boldsymbol{r}_{j},
$$

is a natural "local representation" of the "minimum fluctuation" criterion for electron localization. Figure 1 plots $\zeta_{\sigma}^{R}(\boldsymbol{r})$ for the neon atom. The function is largest in the regions associated with the $1 s$ and $(2 s$, $2 p$ ) orbitals and smaller in the "intershell" region.

One might suspect that a high degree of electron localization would be associated with a small value for the local covariance, as measured by $\zeta_{\sigma}^{h}\left(\boldsymbol{r}_{i}\right)$ or $\zeta_{\sigma}^{R}\left(\boldsymbol{r}_{i}\right)$. Reference to figure 1 establishes that the opposite is in fact true. Taking the volumes of the regions, $\left\{\Omega_{i}\right\}$ towards zero, one forces the local covariance to become rather large because $\sigma$-spin electrons at $\boldsymbol{r}_{i}$ and $\boldsymbol{r}_{i}+\mathrm{d} \boldsymbol{r}_{i}$ are, of course, be highly correlated. Squaring the hole correlation function tends to diminish the importance of regions where $h_{\sigma \sigma}\left(\boldsymbol{r}_{i}, \boldsymbol{r}_{j}\right) \approx 0$ relative to those where $h_{\sigma \sigma}\left(\boldsymbol{r}_{i}, \boldsymbol{r}_{j}\right) \approx-1$. Consequently, a hole that is localized - so that the volume over which its value is close to -1 is relatively large - will be associated with larger values of $\zeta_{\sigma}^{h}\left(\boldsymbol{r}_{i}\right)$ and $\zeta_{\sigma}^{R}\left(\boldsymbol{r}_{i}\right)$ than less localized holes. When the hole correlation function, $h_{\sigma \sigma}\left(\boldsymbol{r}_{i}, \boldsymbol{r}_{j}\right)$, is very compact, its square is even more compact (recall that the

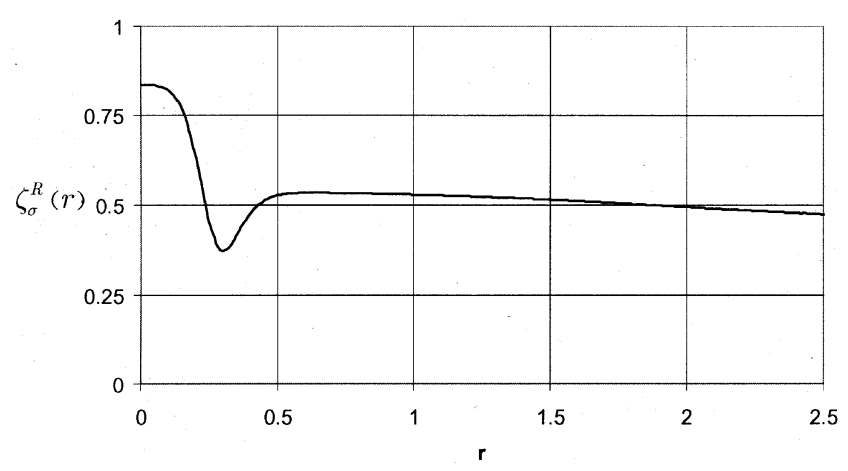

Figure 1. The pointwise minimum correlation per electron, $\zeta_{\sigma}^{R}(\boldsymbol{r})$, for the neon atom (see (36)). 
exchange hole satisfies $\left.-1 \leq h_{\sigma \sigma}\left(\boldsymbol{r}_{i}, \boldsymbol{r}_{j}\right) \leq 0\right)$; indeed, in figure 1 , we observe that

$$
\begin{aligned}
\int \rho\left(\boldsymbol{r}_{j}\right)\left(h\left(\boldsymbol{r}_{i}, \boldsymbol{r}_{j}\right)\right)^{2} \mathrm{~d} \boldsymbol{r}_{j} & =\zeta_{\sigma}^{R}\left(\boldsymbol{r}_{i}\right)<1 \\
& =-\int \rho\left(\boldsymbol{r}_{j}\right) h\left(\boldsymbol{r}_{i}, \boldsymbol{r}_{j}\right) \mathrm{d} \boldsymbol{r}_{j} .
\end{aligned}
$$

More localized holes give larger regions where $\left(h_{\sigma \sigma}\left(\boldsymbol{r}_{i}, \boldsymbol{r}_{j}\right)\right)^{2}$ is appreciable, giving larger values for $\zeta_{\sigma}^{h}\left(\boldsymbol{r}_{i}\right)$ and $\zeta_{\sigma}^{R}\left(\boldsymbol{r}_{i}\right)$. This will become more clear in the following sections, when a specific model for $h_{\sigma \sigma}\left(\boldsymbol{r}_{i}, \boldsymbol{r}_{j}\right)$ is considered.

2.3 The link between the local covariance, $\zeta_{\sigma}^{h}\left(\boldsymbol{r}_{i}\right)$, and the Becke-Edgecombe ELF

The local covariance measure, $\zeta_{\sigma}^{h}\left(\boldsymbol{r}_{i}\right)$ (cf. (26)) is closely linked to the electron localization measure previously proposed by Becke and Edgecombe (cf. (5)). To show this, write (26) using the spherical averaged exchange correlation hole,

$$
h_{\sigma \sigma}^{S A}\left(\boldsymbol{r}_{i}, r_{j}\right) \equiv \frac{1}{4 \pi} \int_{0}^{\pi} \int_{0}^{2 \pi} h_{\sigma \sigma}\left(\boldsymbol{r}_{i}, r_{j}, \theta, \phi\right) \sin (\theta) \mathrm{d} \phi \mathrm{d} \theta,
$$

obtaining

$$
\zeta_{\sigma}^{h}\left[\rho ; \boldsymbol{r}_{i}\right] \equiv \rho_{\sigma}\left(\boldsymbol{r}_{i}\right) \int\left(h_{\sigma \sigma}^{S A}\left(\boldsymbol{r}_{i}, r_{j}\right)\right)^{2} 4 \pi r_{j}^{2} \mathrm{~d} r_{j} .
$$

Suppose we have a model functional, $\mathbf{h}_{\sigma \sigma}^{S A}\left[\rho ; \boldsymbol{r}_{i}, r_{j}\right]$, for the spherically averaged exchange-correlation hole. Since the pairing of electrons is imposed by the Pauli principle, we shall follow the lead of Becke and Edgecombe and model only the exchange hole. Next, suppose that the model functional has a single functional form, so that it takes the form, $\mathbf{h}_{\sigma \sigma}\left(k\left[\rho ; \boldsymbol{r}_{i}\right] r_{j}\right)$, where $k\left[\rho ; \boldsymbol{r}_{i}\right]$ is used to modulate the "width" of the hole. An expression for $k\left[\rho ; \boldsymbol{r}_{i}\right]$ can be deduced from (4). Specifically, from

$$
\begin{aligned}
\partial^{2} \mathbf{h}_{\sigma \sigma}\left(k\left[\rho ; \boldsymbol{r}_{i}\right] r_{j}\right) / \partial r_{j}^{2} & =\partial^{2} \mathbf{h}_{\sigma \sigma}\left(r_{j}\right) / \partial r_{j}^{2}, \\
k^{2}\left[\rho ; \boldsymbol{r}_{i}\right] & =2 \kappa\left[\rho_{\alpha}, \rho_{\beta} ; \boldsymbol{r}_{i}\right],
\end{aligned}
$$

we have

$$
k\left[\rho ; \boldsymbol{r}_{i}\right]=2 k\left[\rho_{\alpha}, \rho_{\beta} ; \boldsymbol{r}_{i}\right]^{1 / 2} / \mathbf{h}^{\prime \prime}(0) .
$$

The notation indicates that $k\left[\rho ; \boldsymbol{r}_{i}\right]$ is a functional of the electron density, $\rho(\boldsymbol{r})$ and a function of the point, $\boldsymbol{r}_{i}$, where we are studying the degree of electron localization. Similarly, $2 k\left[\rho_{\alpha}, \rho_{\beta} ; \boldsymbol{r}_{i}\right]$ is a functional of the spin-densities and a function of the location under investigation.

Substitution of (41) into (39) yields

$$
\begin{aligned}
& \zeta_{\sigma}^{h}\left[\rho ; \boldsymbol{r}_{i}\right] \equiv \rho\left(\boldsymbol{r}_{i}\right) \int_{0}^{\infty}\left(\mathbf{h}_{\sigma \sigma}\left(k\left[\rho ; \boldsymbol{r}_{i}\right] \cdot r_{j}\right)\right)^{2} 4 \pi r_{j}^{2} \mathrm{~d} r_{j} \\
&= \frac{\rho\left(\boldsymbol{r}_{i}\right)}{k\left[\rho ; \boldsymbol{r}_{i}\right]^{3}}\left[\int_{0}^{\infty} \boldsymbol{h}_{\sigma \sigma}^{2}\left(k\left[\rho ; \boldsymbol{r}_{i}\right] r_{j}\right) 4 \pi\left(k\left[\rho ; \boldsymbol{r}_{i}\right] r_{j}\right)^{2}\right. \\
&\left.\times \mathrm{d}\left(k[\rho ; \boldsymbol{x}] r_{j}\right)\right]
\end{aligned}
$$

Using (41), we obtain

$$
\begin{aligned}
\zeta_{\sigma}^{h}\left[\rho ; \boldsymbol{r}_{i}\right]= & \left(\left(\mathbf{h}^{\prime \prime}(0) / 2\right)^{3 / 2} \int_{0}^{\infty} \mathbf{h}_{\sigma \sigma}^{2}(x) 4 \pi x^{2} \mathrm{~d} x\right) \\
& \frac{\rho\left(\boldsymbol{r}_{i}\right)}{\kappa\left[\rho_{\alpha}, \rho_{\beta} ; \boldsymbol{r}_{i}\right]^{3 / 2}} \propto\left(\xi_{\sigma}\left(\boldsymbol{r}_{i}\right)\right)^{-3 / 2}
\end{aligned}
$$

Thus, $\zeta_{\sigma}^{h}\left(\boldsymbol{r}_{i}\right)$, when raised to the $-2 / 3$ rd power, is proportional to the original Becke-Edgecombe measure of electron localization.

The proportionality constant in (43) depends on the model for the hole correlation function. Following Lee and Parr, ${ }^{22}$ we choose a Gaussian model, $\mathbf{h}_{\sigma \sigma}^{G}$ $\left(k\left(\boldsymbol{r}_{i}\right) r_{j}\right) \approx-e^{-\left(k\left(\boldsymbol{r}_{i}\right) r_{j}\right)^{2}}$, for the exchange hole: this choice gives better accuracy for the energy of atomic systems than a similar model based on the uniform electron gas. Substitution of the appropriate results into (43) gives

$$
\begin{aligned}
\zeta_{\sigma}^{h, G}\left[\rho ; \boldsymbol{r}_{i}\right] & \equiv\left(\frac{\pi}{2}\right)^{3 / 2} \frac{\rho_{\sigma}\left(\boldsymbol{r}_{i}\right)}{\kappa_{\sigma}^{B E}\left[\rho_{\alpha}, \rho_{\beta} ; \boldsymbol{r}_{i}\right]^{3 / 2}} \\
& =\left(\frac{3 \pi}{4}\right)^{3 / 2} \frac{\rho_{\sigma}\left(\boldsymbol{r}_{i}\right)^{5 / 2}}{\left[\tau_{\sigma}\left(\boldsymbol{r}_{i}\right)-\tau_{\sigma}^{W}\left(\boldsymbol{r}_{i}\right)\right]^{3 / 2}}
\end{aligned}
$$

In figure 2 the Gaussian model for $\zeta_{\sigma}^{h}\left(\boldsymbol{r}_{i}\right),(44)$, is plotted for the Neon atom.

When plotting electron localization functions, Becke and Edgecombe found it prudent to plot not the "raw" electron localization measure, but instead a function of the index, ${ }^{11}$

$$
\lambda_{\sigma}^{B E}\left(\boldsymbol{r}_{i}\right) \equiv \frac{1}{1+\xi_{\sigma}^{2}\left(\boldsymbol{r}_{i}\right)} \sim \frac{1}{1+\zeta_{\sigma}^{-2}\left(\boldsymbol{r}_{i}\right)} .
$$

This author has found, however, that transformation 


$$
\begin{aligned}
\mathrm{L}_{\sigma}\left(\boldsymbol{r}_{i}\right) \equiv \tanh \left(\xi_{\sigma}^{-1}\left(\boldsymbol{r}_{i}\right)-\xi_{\sigma}\left(\boldsymbol{r}_{i}\right)\right) \\
\sim \tanh \left(\zeta_{\sigma}\left(\boldsymbol{r}_{i}\right)-\zeta_{\sigma}^{-1}\left(\boldsymbol{r}_{i}\right)\right)
\end{aligned}
$$

is often even more effective in bringing out the essential structure of an electron localization measure. (In (45)-(46) we have indicated that analogous transformations for the local covariances, $\zeta_{\sigma}\left(\boldsymbol{r}_{i}\right)$, can be obtained by substituting $\zeta_{\sigma}^{-1}\left(\boldsymbol{r}_{i}\right)$ for $\xi_{\sigma}\left(\boldsymbol{r}_{i}\right)$.) Figure 3 compares the properties of these transformations for the Becke-Edgecombe ELF,

$$
\xi_{\sigma}^{B E}\left(\boldsymbol{r}_{i}\right) \equiv \frac{10}{3\left(6 \pi^{2}\right)^{2 / 3}} \frac{\tau_{\sigma}\left(\boldsymbol{r}_{i}\right)-\tau_{\sigma}^{W}\left(\boldsymbol{r}_{i}\right)}{\rho_{\sigma}{ }^{5 / 3}\left(\boldsymbol{r}_{i}\right)}
$$

revealing the superiority of the hyperbolic tangent form. Even more general than the hyperbolic tangent form, we can consider a measure such as

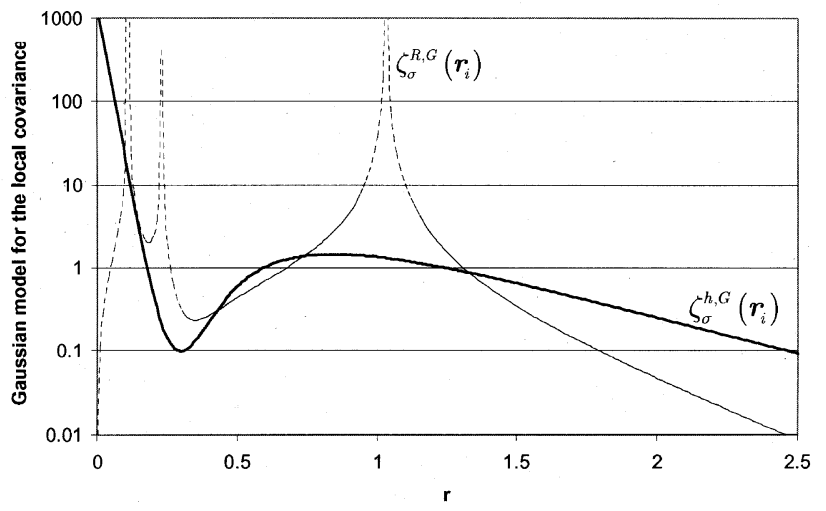

Figure 2. The Gaussian model for the local covariances, $\zeta_{\sigma}^{h, G}\left(\boldsymbol{r}_{i}\right)(44)$, and $\zeta_{\sigma}^{R, G}\left(\boldsymbol{r}_{i}\right)(62)$ of the neon atom.

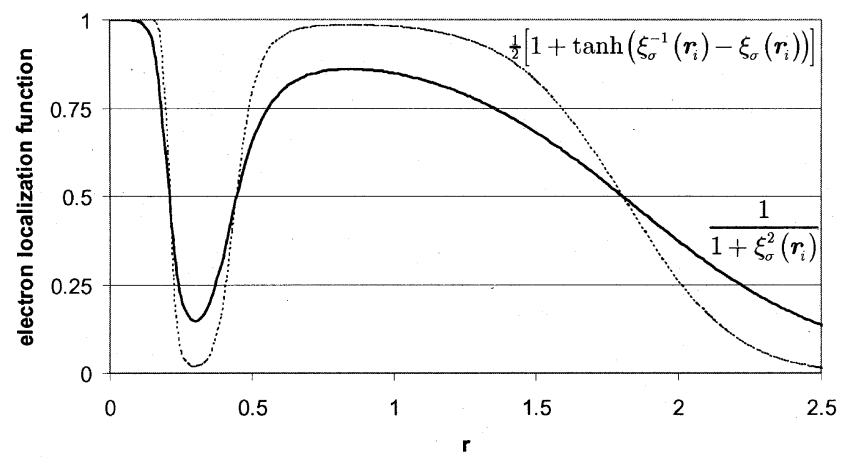

Figure 3. Comparison of the Becke-Edgecombe form, (45), and the hyperbolic tangent form, (46), of the electron localization function for the neon atom. For comparison purposes, the hyperbolic tangent form is mapped onto the unit interval.

$$
\begin{aligned}
\mathrm{L}_{\sigma}\left(\boldsymbol{r}_{i}\right) \equiv \tanh \left(\xi_{\sigma}^{-k}\left(\boldsymbol{r}_{i}\right)-\xi_{\sigma}^{k}\left(\boldsymbol{r}_{i}\right)\right) \\
\sim \tanh \left(\zeta_{\sigma}^{k}\left(\boldsymbol{r}_{i}\right)-\zeta_{\sigma}^{-1}\left(\boldsymbol{r}_{i}\right)\right)
\end{aligned}
$$

where $k \geq 1$. Larger values of $k$ make the shell structure even more apparent than the simplest $k=1$ case.

Figure 4 examines what occurs if we use a model other than the uniform electron gas as the "reference value" in the denominator of $\xi_{\sigma}^{B E}\left(\boldsymbol{r}_{i}\right)$. Specifically, for the nearly free electron gas, we would have

$$
\xi_{\sigma}^{\text {grad }}\left(\boldsymbol{r}_{i}\right) \equiv \frac{\tau_{\sigma}\left(\boldsymbol{r}_{i}\right)-\tau_{\sigma}^{W}\left(\boldsymbol{r}_{i}\right)}{\tau_{\sigma}^{\text {grad }}\left(\boldsymbol{r}_{i}\right)-\tau_{\sigma}^{W}\left(\boldsymbol{r}_{i}\right)}
$$

where $\tau_{\sigma}^{\text {grad }}\left(\boldsymbol{r}_{i}\right)$ is the local kinetic energy that results from the gradient expansion, (65). This form reduces to (47) for the uniform electron gas, but would be expected to provide a better description for systems with nonuniform electron density. However, the results are much less satisfactory. This is somewhat counterintuitive, since one might suppose that using a superior model for "reference value" would improve the quality of our results. The fact the model deteriorates for improved reference systems suggests that the significance of the $\rho^{5 / 3}\left(\boldsymbol{r}_{i}\right)$ dependence in the denominator of (5) might be better understood through the local covariance argument presented here or one of the other reference-free arguments available in the literature. ${ }^{26,27}$

\subsection{A measure of electron localization based on (36)}

We can also develop a measure of electron localization based on $\zeta_{\sigma}^{R}\left(\boldsymbol{r}_{i}\right)$ (cf. (36)). To do this, we change the coordinates of integration $\boldsymbol{s}=\boldsymbol{r}_{j}-\boldsymbol{r}_{i}$,

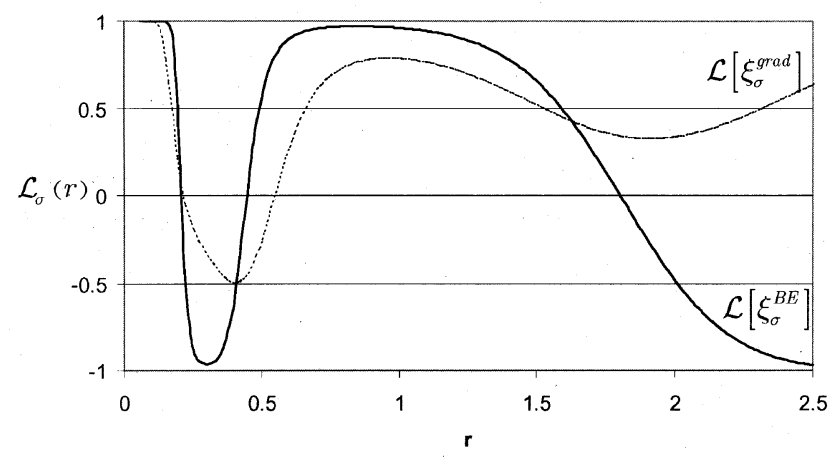

Figure 4. Dependence of the Becke-Edgecombe electron localization function on the choice of reference kinetic energy density. $\mathrm{L}_{\sigma}(r)$ (cf. (46)) is plotted for $\xi_{\sigma}^{B E}(\boldsymbol{r})$, (47), and the form using the gradient-corrected kineticenergy function, $\xi_{\sigma}^{\text {grad }}(\boldsymbol{r})$, (49). 


$$
\begin{gathered}
\zeta_{\sigma}^{R}\left(\boldsymbol{r}_{i}\right)=\int_{0} \rho_{\sigma}\left(\boldsymbol{r}_{i}+\boldsymbol{s}\right) h_{\sigma \sigma}^{2}\left(\boldsymbol{r}_{i}, \boldsymbol{r}_{i}+\boldsymbol{s}\right) \mathrm{d} \boldsymbol{s} \\
=\left\{\int_{0}^{\infty}\left[\frac{1}{4 \pi} \int_{0}^{\pi} \int_{0}^{2 \pi} \rho_{\sigma}\left(\boldsymbol{r}_{i}+\boldsymbol{s}\right) h_{\sigma \sigma}^{2}\left(\boldsymbol{r}_{i}, \boldsymbol{r}_{i}+\boldsymbol{s}\right) \sin (\theta) \mathrm{d} \theta \mathrm{d} \phi\right]\right. \\
\left.\times 4 \pi s^{2} \mathrm{~d} s\right\}
\end{gathered}
$$

Integrals with the form of the innermost integral are evaluated using the general formula ${ }^{19}$

$$
\begin{aligned}
\frac{1}{4 \pi} \int_{0}^{\pi} \int_{0}^{2 \pi} f\left(\boldsymbol{r}_{i}, \boldsymbol{s}\right) \sin (\boldsymbol{\theta}) \mathrm{d} \theta \mathrm{d} \phi & \\
= & {\left[\left(1+\frac{s^{2}}{3 !} \nabla_{j}^{2}+\frac{s^{4}}{5 !} \nabla_{j}^{4}+\cdots\right) f\left(\boldsymbol{r}_{i}, \boldsymbol{r}_{j}\right)\right]_{r_{i}=\boldsymbol{r}_{j}}, }
\end{aligned}
$$

by which we attain, for (50),

$$
\begin{gathered}
\zeta_{\sigma}^{R}\left(\boldsymbol{r}_{i}\right)=\int \rho_{\sigma}\left(\boldsymbol{r}_{i}+\boldsymbol{s}\right) h_{\sigma \sigma}^{2}\left(\boldsymbol{r}_{i}, \boldsymbol{r}_{i}+\boldsymbol{s}\right) \mathrm{d} \boldsymbol{s} \\
=\rho\left(\boldsymbol{r}_{i}\right)\left\{\int_{0}^{\infty}\left[1+s^{2}\left(\frac{8 \tau_{\sigma}^{W}\left(\boldsymbol{r}_{i}\right)-8 \tau_{\sigma}\left(\boldsymbol{r}_{i}\right)+\nabla^{2} \rho\left(\boldsymbol{r}_{i}\right)}{6 \rho\left(\boldsymbol{r}_{i}\right)}\right)+\cdots\right]\right. \\
\left.\times 4 \pi s^{2} \mathrm{~d} s\right\} .
\end{gathered}
$$

Just as in the previous section, we assume that we have a model function for the integrand in (52), so that

$$
\zeta_{\sigma}^{R}\left(\boldsymbol{r}_{i}\right)=\rho\left(\boldsymbol{r}_{i}\right) \int_{0}^{\infty} f\left(\boldsymbol{r}_{i}, l\left[\rho, \boldsymbol{r}_{i}\right] s\right) 4 \pi s^{2} \mathrm{~d} s,
$$

where $l\left[\rho ; \boldsymbol{r}_{i}\right]$ is a functional of the electron density and a function of the position of the reference electron. As before, we can write

$$
\begin{aligned}
\zeta_{\sigma}^{R}\left(\boldsymbol{r}_{i}\right)= & \frac{\rho\left(\boldsymbol{r}_{i}\right)}{\left(l\left[\rho, \boldsymbol{r}_{i}\right]\right)^{3}} \\
& \quad \times \int_{0}^{\infty} f\left(\boldsymbol{r}_{i}, l\left[\rho, \boldsymbol{r}_{i}\right] s\right) 4 \pi\left(l\left[\rho, \boldsymbol{r}_{i}\right] s\right)^{2} \mathrm{~d}\left(l\left[\rho, \boldsymbol{r}_{i}\right] s\right) \\
= & \frac{\rho\left(\boldsymbol{r}_{i}\right)}{\left(l\left[\rho, \boldsymbol{r}_{i}\right]\right)^{3}} \int_{0}^{\infty} f\left(\boldsymbol{r}_{i}, x\right) 4 \pi x^{2} \mathrm{~d} x
\end{aligned}
$$

and

$$
\left.\frac{\partial^{2} f\left(\boldsymbol{r}_{i}, l\left[\rho, \boldsymbol{r}_{i}\right] s\right)}{\partial s^{2}}\right|_{s=0}=\left.\frac{\partial^{2} f\left(\boldsymbol{r}_{i}, s\right)}{\partial s^{2}}\right|_{s=0}\left(l\left[\rho, \boldsymbol{r}_{i}\right]\right)^{2}
$$

so that, from (52),

$$
\begin{aligned}
l\left[\rho, \boldsymbol{r}_{i}\right]= & \left(\left(\left.\frac{\partial^{2} f\left(\boldsymbol{r}_{i}, s\right)}{\partial s^{2}}\right|_{s=0}\right)^{-1}\right. \\
& \left.\times \frac{8 \tau_{\sigma}^{W}\left(\boldsymbol{r}_{i}\right)-8 \tau_{\sigma}\left(\boldsymbol{r}_{i}\right)+\nabla^{2} \rho\left(\boldsymbol{r}_{i}\right)}{3 \rho\left(\boldsymbol{r}_{i}\right)}\right)^{1 / 2} .
\end{aligned}
$$

From this we infer that

$$
\begin{aligned}
\zeta_{\sigma}^{R}\left(\boldsymbol{r}_{i}\right) & \propto \rho\left(\boldsymbol{r}_{i}\right) /\left(\frac{8 \tau_{\sigma}\left(\boldsymbol{r}_{i}\right)-8 \tau_{\sigma}^{W}\left(\boldsymbol{r}_{i}\right)-\nabla^{2} \rho\left(\boldsymbol{r}_{i}\right)}{3 \rho\left(\boldsymbol{r}_{i}\right)}\right)^{3 / 2} \\
& \propto \frac{3^{3 / 2} \rho^{5 / 2}\left(\boldsymbol{r}_{i}\right)}{\left(8 \tau_{\sigma}\left(\boldsymbol{r}_{i}\right)-8 \tau_{\sigma}^{W}\left(\boldsymbol{r}_{i}\right)-\nabla^{2} \rho\left(\boldsymbol{r}_{i}\right)\right)^{3 / 2}}
\end{aligned}
$$

In writing this equation, we assume that $\left.\frac{\partial^{2} f\left(\boldsymbol{r}_{i}, s\right)}{\partial s^{2}}\right|_{s=0}<0$. When the coefficient of $s^{2}$ in (52) is not negative, the simplistic treatment used here is insufficient to characterize the function and, problematically, the denominator in (57) is imaginary. In order to ensure that (57) is always a real-valued functional of the electron density, we introduce the absolute value into the denominator to obtain the working formula,

$$
\zeta_{\sigma}^{R}\left(\boldsymbol{r}_{i}\right) \propto \frac{3^{3 / 2} \rho^{5 / 2}\left(\boldsymbol{r}_{i}\right)}{\left|8 \tau_{\sigma}\left(\boldsymbol{r}_{i}\right)-8 \tau_{\sigma}^{W}\left(\boldsymbol{r}_{i}\right)-\nabla^{2} \rho\left(\boldsymbol{r}_{i}\right)\right|^{3 / 2}} .
$$

To determine the constant of proportionality in (57), we need to consider a model for $f\left(\boldsymbol{r}_{i}, l\left[\rho, \boldsymbol{r}_{i}\right] s\right)$. Again as before, we consider a Gaussian model, with

$$
f^{G}\left(\boldsymbol{r}_{i}, l\left[\rho, \boldsymbol{r}_{i}\right] s\right)=e^{-\left(l\left[\rho ; \boldsymbol{r}_{i}\right] s\right)^{2}} .
$$

Within the confines of this model, we have that

$$
\int_{0}^{\infty} f^{G}\left(\boldsymbol{r}_{i}, x\right) 4 \pi x^{2} \mathrm{~d} x=\pi^{3 / 2}
$$

and

$$
\left.\frac{\partial^{2} f^{G}\left(\boldsymbol{r}_{i}, s\right)}{\partial s^{2}}\right|_{s=0}=-2
$$

and so, from (54),

$$
\begin{aligned}
\zeta_{\sigma}^{R, G}\left(\boldsymbol{r}_{i}\right) & =\frac{(3 \pi)^{3 / 2} \rho^{5 / 2}\left(\boldsymbol{r}_{i}\right)}{\left|4 \tau_{\sigma}\left(\boldsymbol{r}_{i}\right)-4 \tau_{\sigma}^{W}\left(\boldsymbol{r}_{i}\right)-\frac{1}{2} \nabla^{2} \rho\left(\boldsymbol{r}_{i}\right)\right|^{3 / 2}} \\
& =\left(\frac{3 \pi}{4}\right)^{3 / 2} \frac{\rho^{5 / 2}\left(\boldsymbol{r}_{i}\right)}{\left|\tau_{\sigma}\left(\boldsymbol{r}_{i}\right)-\tau_{\sigma}^{W}\left(\boldsymbol{r}_{i}\right)-\frac{1}{8} \nabla^{2} \rho\left(\boldsymbol{r}_{i}\right)\right|^{3 / 2}} .
\end{aligned}
$$


The Gaussian model for $\zeta_{\sigma}^{R}\left(\boldsymbol{r}_{i}\right)$, given by (62), is plotted in figure 2 .

Superficial observation of $\zeta_{\sigma}^{R, G}\left(\boldsymbol{r}_{i}\right)$ reveals that the shell structure in the Gaussian model is even more pronounced than it is in the true function, $\zeta_{\sigma}^{R}\left(\boldsymbol{r}_{i}\right)$. Part the discrepancy may be blamed on imperfections in the Gaussian model, which gives a localized hole. (The true exchange hole is significantly delocalized.) In addition, the superiority of the approximate result to the exact result may be attributed to the fact higher-order terms in the Taylor series expansion for the exchange hole (cf. (4)) can obscure the decisiveness of the quadratic term in describing the relative extent of electron localization. By relying only on the quadratic term, the present model provides a clear indication of the relative amount of electron localization.

In figure 5, we plot the electron localization measures, $\mathrm{L}_{\sigma}\left[\zeta_{\sigma}^{h, G}\right]$ and $\mathrm{L}_{\sigma}\left[\zeta_{\sigma}^{R, G}\right]$ corresponding to (44) and (62), respectively. The form using the local representation of the correlation matrix, $\zeta_{\sigma}^{h, G}\left(\boldsymbol{r}_{i}\right)$, is particularly effective at distinguishing the shell structure of the Neon atom. The largest difficulty arises because of the divergence of the Laplacian near the atomic nucleus. If one keeps this in mind and neglects points very near the atomic nucleus, then there is no difficulty in clearly distinguishing the shell structure from $\mathrm{L}_{\sigma}\left[\zeta_{\sigma}^{R, G}\right]$. (Another alternative is to remove the singularity of the Laplacian by introducing a weight function; the next section provides insight into how this may be accomplished.)

Unsurprisingly, given the link between $\zeta_{\sigma}^{h, G}\left(\boldsymbol{r}_{i}\right)$ and the conventional electron localization function (recall that $\left.\zeta_{\sigma}^{h, G}\left(\boldsymbol{r}_{i}\right) \propto\left(\xi_{\sigma}\left(\boldsymbol{r}_{i}\right)\right)^{-3 / 2}\right), \mathrm{L}_{\sigma}\left[\zeta_{\sigma}^{h, G}\right]$ is also an effective measure of electron localization. The

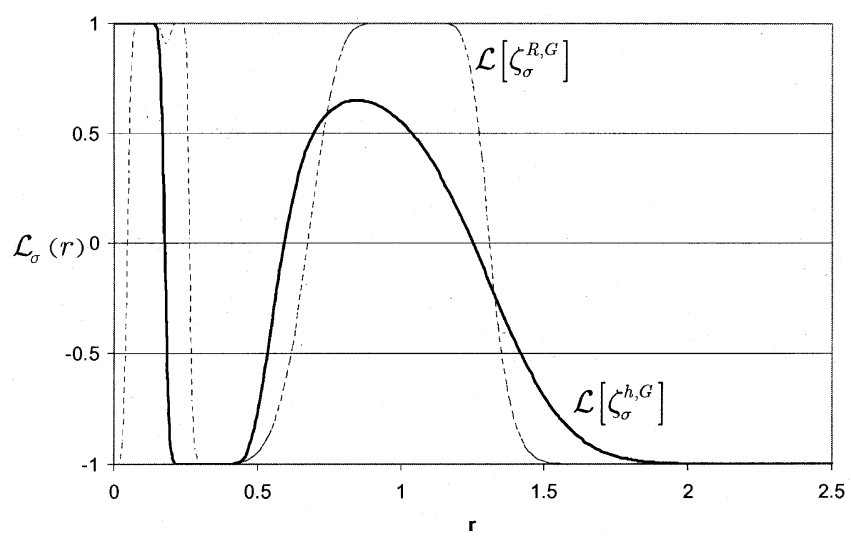

Figure 5. Electron-localization functions based on the local covariances, $\zeta_{\sigma}^{h, G}\left(\boldsymbol{r}_{i}\right)(44)$, and $\zeta_{\sigma}^{R, G}\left(\boldsymbol{r}_{i}\right)(62)$, of the neon atom. fluctuations in this localization measure are less pronounced than those when the "raw" BeckeEdgecombe measure, $\xi_{\sigma}\left(\boldsymbol{r}_{i}\right)$ is used instead. This can be understood from the perspective of (48): raising the electron localization measure to a power, $k$, with $|k|>1$, tends to enhance the shell structure.

\subsection{Computing ELFs without using orbitals}

The most time-consuming step in evaluating these measures of electron localization is the determination of the local kinetic energy, $\tau_{\sigma}\left(\boldsymbol{r}_{i}\right)$, which depends on the Kohn-Sham orbitals for a given system. While there are many methods for obtaining the Kohn-Sham orbitals from the electron density (as computed from computational chemistry or measured by X-ray spectroscopy), ${ }^{29-32}$ it is reasonable to wonder whether we might avoid computing the orbitals. One way to do this is to consider using an approximate kinetic energy functional computed using the gradient expansion. ${ }^{33-35}$

In figure 6 we consider several different approximate expressions for the kinetic-energy, including the Thomas-Fermi form,

$$
\tau_{\sigma}^{T F}(\boldsymbol{r}) \equiv(3 / 10)\left(6 \pi^{2}\right)^{2 / 3} \rho_{\sigma}^{5 / 3}(\boldsymbol{r})
$$

the Weisacker expression,

$$
\tau_{\sigma}^{W}(\boldsymbol{r})=(1 / 8)\left(\nabla \sqrt{\rho_{\sigma}(\boldsymbol{r})} \cdot \nabla \sqrt{\rho_{\sigma}(\boldsymbol{r})}\right),
$$

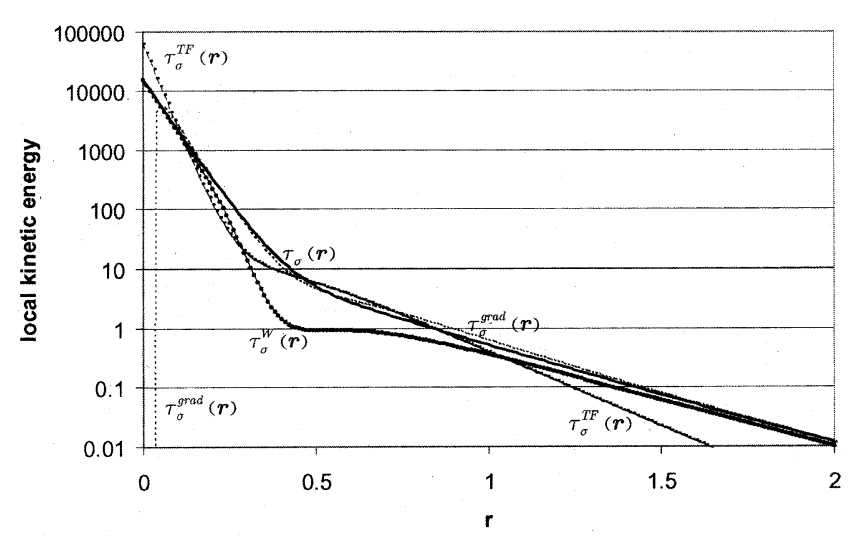

Figure 6. Models for the local kinetic energy including the Thomas-Fermi model, $\tau_{\sigma}^{T F}(r)$, Weisacker model, $\tau_{\sigma}^{W}(r)$, and the gradient expansion $\tau_{\sigma}^{\text {grad }}(r)$. These models are computed for the neon atom, and compared to the accurate kinetic energy density, $\tau_{\sigma}(r)$, which is denoted with a heavy solid line. 
and the conventional gradient expansion,

$$
\tau_{\sigma}^{\text {grad }}(\boldsymbol{r})=\tau_{\sigma}^{T F}(\boldsymbol{r})+(1 / 9) \tau_{\sigma}^{W}(\boldsymbol{r})+(1 / 6) \nabla^{2} \rho_{\sigma}(\boldsymbol{r})
$$

As previously noted by Tsirelson and coworkers, the gradient expression is rather accurate except in regions very near the nucleus, where the Laplacian of the electron density,

$$
\nabla^{2} \rho_{\sigma}(\boldsymbol{r})=\frac{\partial^{2} \rho(\boldsymbol{r})}{\partial r^{2}}-\frac{2}{r} \frac{\partial \rho(\boldsymbol{r})}{\partial r}
$$

diverges. Near the nucleus, however, only the atomic $1 s$ orbital contributes to the kinetic energy, so the Weisacker term is rather accurate. This suggests that we consider a form of the kinetic energy like

$$
t_{\sigma}^{\bmod }(\boldsymbol{r})=w(\boldsymbol{r}) t_{\sigma}^{W}(\boldsymbol{r})+(1-w(\boldsymbol{r})) t_{\sigma}^{\text {grad }}(\boldsymbol{r})
$$

where $w(\boldsymbol{r})$ is a weight function that is close to one near atomic nuclei, and small elsewhere. Based on the fact that the $1 s$ orbital of the one-electron atom with atomic number $Z$ falls off to half its value at the nucleus at

$$
r_{1 / 2}=\ln (2) / Z
$$

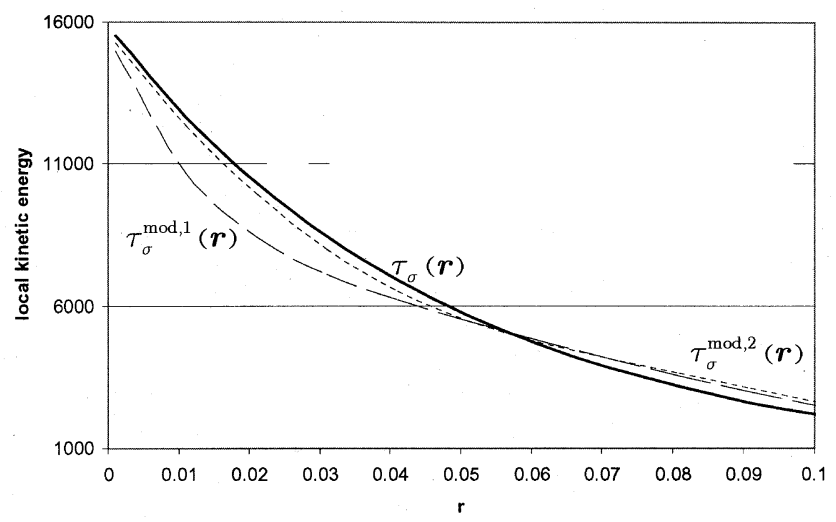

Figure 7. Models for the kinetic energy density based on switching from the kinetic energy density of the gradient expansion to the Weisacker kinetic energy density using $w_{1}(\boldsymbol{r})(69)$ and $w_{2}(\boldsymbol{r})(70)$. The form of the kinetic energy density is computed using (67) and denoted $\tau_{\sigma}^{\text {mod, }}(\boldsymbol{r})$ and $\tau_{\sigma}^{\text {mod,2 }}(\boldsymbol{r})$ according to whether $w_{1}(\boldsymbol{r})$ or $w_{2}(\boldsymbol{r})$ is employed. Results are compared to the accurate kinetic energy density of the neon atom, $\tau(\boldsymbol{r})$. Outside the range plotted here, dependence on the weight function is almost indistinguishable. we expect that for values of $r$ greater than $r_{1 / 2}$ the gradient expansion will be satisfactory, while for smaller values of $r$ the $1 s$ orbital is dominant and the Weisacker form should be acceptable. We consider two possible forms for the "switching function" $w(\boldsymbol{r})$,

$$
\begin{aligned}
& w_{1}(\boldsymbol{r})=e^{-\ln (2)(Z r / \ln (2))^{2}} \\
& w_{2}(\boldsymbol{r})=e^{-\ln (2)(Z r / \ln (2))^{4}} .
\end{aligned}
$$

Both weight functions are equated to $\frac{1}{2}$ when $r=r_{1 / 2}$, and so both functions switch from the Weisacker form to the gradient expansion in a similar way. As seen in figure 7, the latter form, (70), is superior for canceling out the singularity in the Laplacian, while the former expression is arguably simpler and more intuitive.

Tsirelson and coworkers have already demonstrated that the kinetic energy density from the gradient expansion is accurate enough to recover the electron localization function except in the immediate vicinity of the nucleus. It is unsurprising, then, that the present "correction" to the kinetic-energy density (using $w_{2}(\boldsymbol{r})$ in (67); see figure 8) also reproduces the electronic shell structure. The shell structure is less pronounced, however, when the approximate kinetic-energy density is used, though the shell structure is still decisively apparent when $\zeta_{\sigma}^{R, G}\left(\boldsymbol{r}_{i}\right)$ is considered.

This generally favorable result suggests that we could approximate the kinetic-energy density of a molecule with an expression of the form (67), where

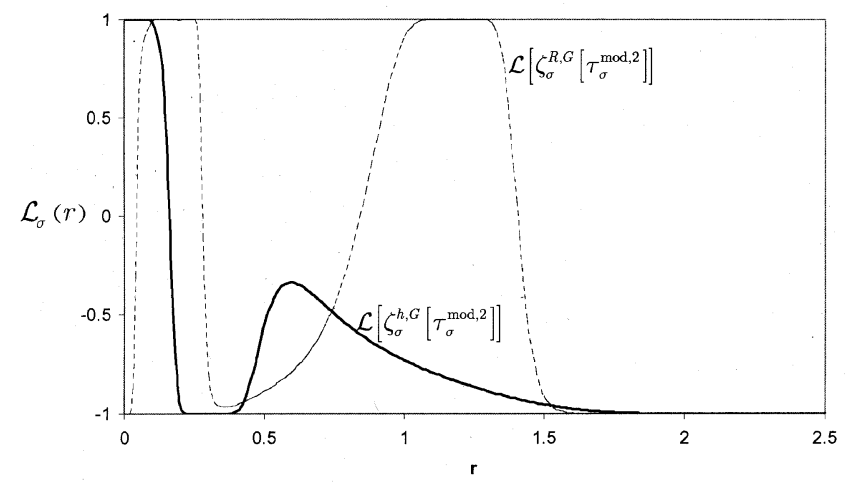

Figure 8. Electron-localization functions based on the local covariances, $\zeta_{\sigma}^{h, G}\left(\boldsymbol{r}_{i}\right)(44)$, and $\zeta_{\sigma}^{R, G}\left(\boldsymbol{r}_{i}\right)$ (62), of the neon atom. Unlike figure 5 , here the covariances are computed using the approximate kinetic-energy density defined using (67) and (70). 


$$
w(\boldsymbol{r})=\sum_{\alpha} e^{-\ln (2)\left(Z_{\alpha}\left|\boldsymbol{r}-\boldsymbol{R}_{\alpha}\right| / \ln (2)\right)^{4}}
$$

Here, $\left\{Z_{\alpha}\right\}$ and $\left\{\boldsymbol{R}_{\alpha}\right\}$ denote the nuclear charges and positions. While $w_{2}(\boldsymbol{r})$ will be greater than one near the nuclei of polyatomic systems, the extreme locality of the quartic form dictates that this will not be problematic for any reasonable nuclear configuration. (Similarly, we expect no problems if a Gaus-
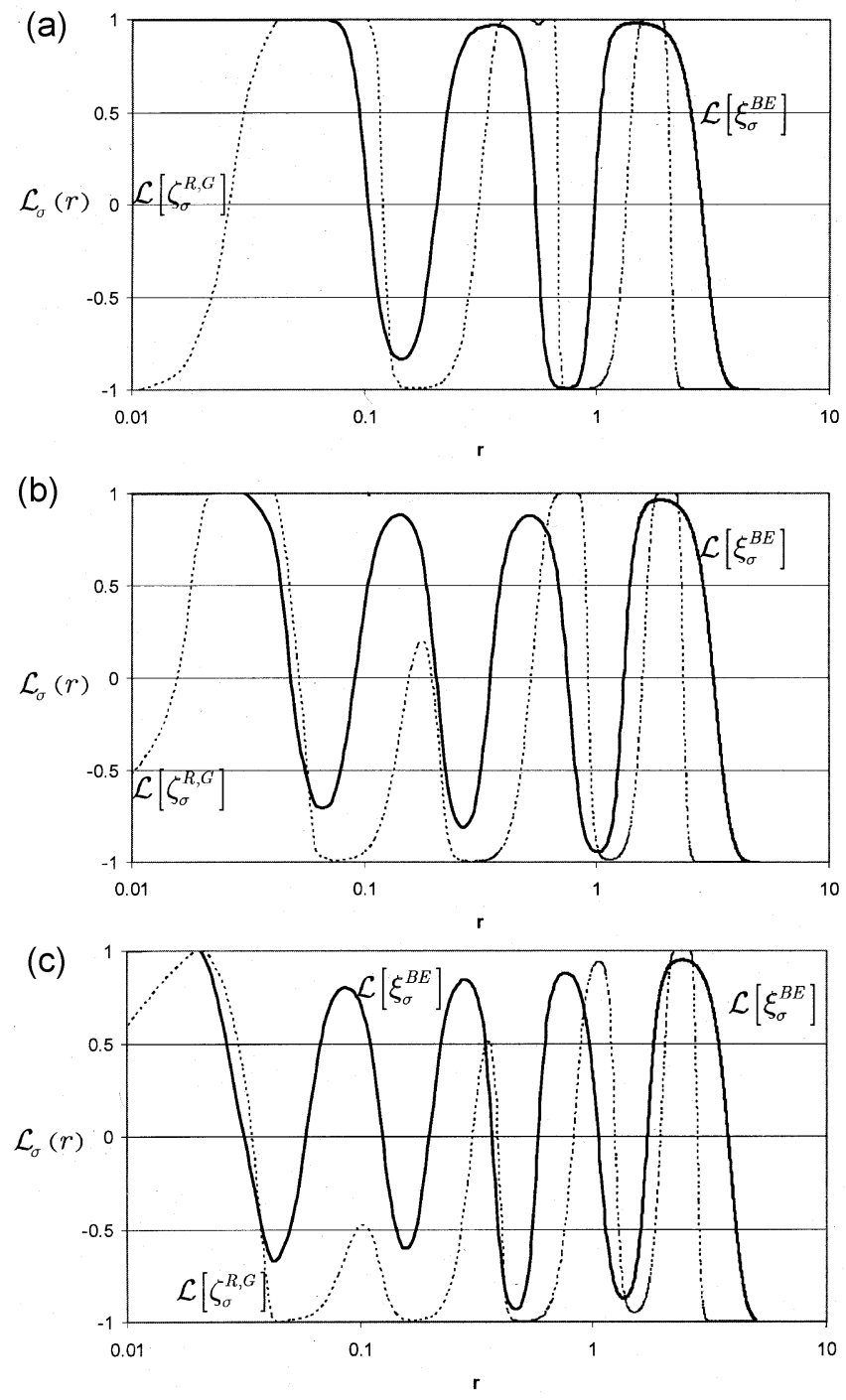

Figure 9. The electron-localization function of Becke and Edgecombe, $\xi_{\sigma}^{B E}\left(\boldsymbol{r}_{i}\right)$, and the local covariance, $\zeta_{\sigma}^{R, G}\left(\boldsymbol{r}_{i}\right)$ (62), are plotted for the (a) argon, (b) krypton, and (c) xenon atoms. The hyperbolic tangent transformation, (46), is employed. A logarithmic scale is used to elucidate shell structure near the atomic cores. The dotted trace corresponds to the transformed local covariance ( $\mathrm{L}\left[\zeta_{\sigma}^{R, G}\right]$ ) and the solid trace corresponds to the transformed Becke-Edgecombe localization measure $\left(L\left[\xi_{\sigma}^{B E}\right]\right)$. sian form, analogous to (69), were used in (71).) Finally, we mention that if we multiply the Laplacian term in $\zeta_{\sigma}^{R, G}\left(\boldsymbol{r}_{i}\right)$ by $1-w(\boldsymbol{r})$, then the anomalous behavior of this local covariance near atomic nuclei is removed. (If this modification is made, then $\zeta_{\sigma}^{R, G}\left(\boldsymbol{r}_{i}\right) \approx \zeta_{\sigma}^{h, G}\left(\boldsymbol{r}_{i}\right)$ in any region where $w(\boldsymbol{r}) \approx 1$.)

\subsection{Extensibility to other systems}

The preceding five sections are intended to elucidate the theoretical foundations of electron localization functions and, in particular, the approach to electron localization functions through local measures of the covariance. Motivated largely by the importance of first and second-row atoms to chemistry, the neon atom was used as a pedagogical example of the key concepts. It remains to be seen, though, to what extent the results are extensible to the remainder of the periodic table. Figures 9 and 10 address this issue.

Figure 9 shows that, even for heavier atoms, the localization indicator based on the correlation matrix, $\zeta_{\sigma}^{R, G}\left(\boldsymbol{r}_{i}\right)$, works very effectively. Indeed, in the valence regions of the molecule, indicators based on $\zeta_{\sigma}^{R, G}\left(\boldsymbol{r}_{i}\right)$ seem to work better than those based on the usual Becke-Edgecombe analysis. The opposite is true near the atomic nuclei. Based on the overweening importance of valence electrons in chemistry, further studies of the localization measure,

$$
\mathrm{L}_{\sigma}\left[\zeta_{\sigma}^{R, G}\right] \equiv \tanh \left(\zeta_{\sigma}^{R, G}\left(\boldsymbol{r}_{i}\right)-\left(\zeta_{\sigma}^{R, G}\left(\boldsymbol{r}_{i}\right)\right)^{-1}\right)
$$

seem warranted. The main difference between localization measures based on the local covariance, $\zeta_{\sigma}^{R, G}\left(\boldsymbol{r}_{i}\right)$, and the Becke-Edgecombe analysis, $\zeta_{\sigma}^{B E}\left(\boldsymbol{r}_{i}\right)$, is that the peaks in the shell structure from the local covariance seem to be shifted outward with respect to those from $\zeta_{\sigma}^{B E}\left(\boldsymbol{r}_{i}\right)$. However, the qualitative picture arising from the two measures of electron localization is, as expected, similar.

The author also explored whether the corrected local kinetic energy defined by (67) and (71) was accurate for heavier atoms. Indeed it is: the weight function in (71) seems to be appropriate at least for the systems studied here ( $\mathrm{Ne}, \mathrm{Ar}, \mathrm{Kr}, \mathrm{Xe})$, and based on this is likely appropriate throughout the periodic table. As was already observed for the neon atom, electron localization measures using the approximate kinetic energy density based on the gradient expansion are, in general, less accurate than those using the accurate kinetic-energy density. (See figure 10.) However, it seems clear that approximate 
kinetic-energy densities are adequate for determining electron localization functions. (This seems to be especially true in the chemically important valence regions where, unsurprisingly, the electron density is varying more slowly and so the gradient expansion is more accurate.)
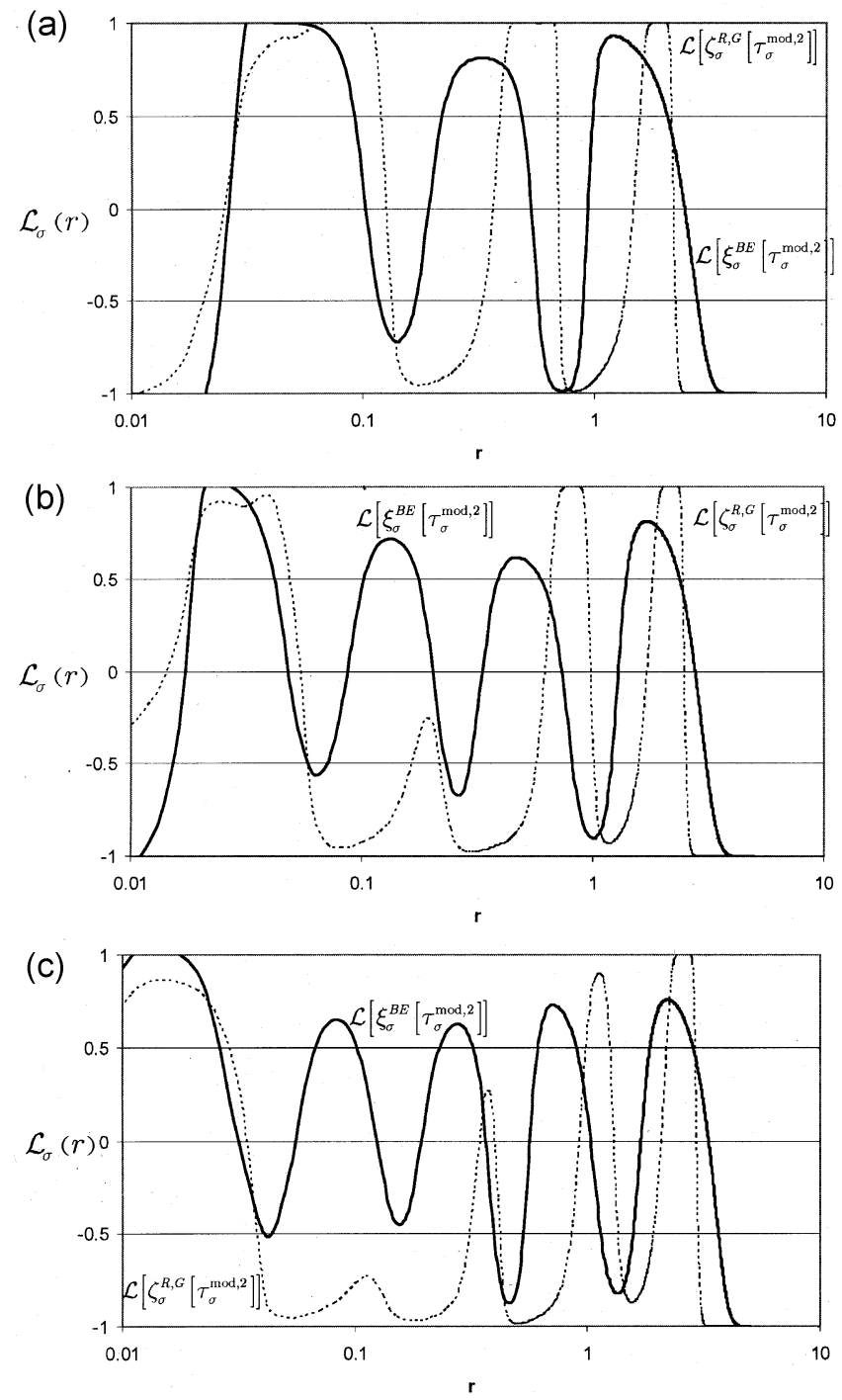

Figure 10. The electron-localization function of Becke and Edgecombe, $\xi_{\sigma}^{B E}\left(\boldsymbol{r}_{i}\right)$, and the local covariance, $\zeta_{\sigma}^{R, G}\left(\boldsymbol{r}_{i}\right)$ (62), are plotted for the (a) argon, (b) krypton, and (c) xenon atoms. The hyperbolic tangent transformation, (46), is employed. A logarithmic scale is used to elucidate shell structure near the atomic cores. Unlike figure 9 , here the covariances are computed using the $a p$ proximate kinetic-energy density defined using (67) and (70). The dotted trace corresponds to the transformed local covariance $\left(\mathrm{L}\left[\zeta_{\sigma}^{R, G}\right]\right)$ and the solid trace corresponds to the transformed Becke-Edgecombe localization measure $\left(L\left[\xi_{\sigma}^{B E}\right]\right)$.

\section{Summary}

The primary contribution of this paper is to show that the electron localization function of Becke and Edgecombe, $\zeta_{\sigma}^{B E}\left(\boldsymbol{r}_{i}\right),(5)$, is related to the magnitude of the local covariance, as expressed through $\zeta_{\sigma}^{h}\left(\boldsymbol{r}_{i}\right)$ (cf. (43)). Here, the local covariance measures the correlation between electrons at $\boldsymbol{r}_{i}$ and other points, $\boldsymbol{r}_{j}$. (The local covariance is computed by considering the covariance, $\sigma_{i j}^{2}$, between the number of electrons in distinct regions of the molecule, $\Omega_{i}$ and $\Omega_{j}$, and then allowing the number of regions to approach infinity and the volume of the regions to approach zero.) Equivalently, $\zeta_{\sigma}^{h}\left(\boldsymbol{r}_{i}\right)$ measures the fluctuation in the number of electrons at the point $\boldsymbol{r}_{i}$. These considerations establish the link between the electron localization measure of Becke and Edgecombe and the venerable studies of Daudel. Significantly, addressing the covariance obviates the need to invoke the existence of a "reference system" when deriving measures for electron localization.

A secondary contribution is the consideration of the correlation matrix, $R_{i j}=\sigma_{i j}^{2} / \sqrt{\sigma_{i i}^{2} \sigma_{j j}^{2}}$. In the limit of infinitesimal regions, we again acquire a local measure of the covariance, denoted $\zeta_{\sigma}^{R}\left(\boldsymbol{r}_{i}\right)$. For the systems considered here, this index seems to perform better than other measures of electron localization in the chemically important valence regions of atoms. It is not entirely clear why this is so, but one may theorize that this measure combines terms normally associated with electron localization function (notably, the difference between the kinetic energy density and the Weisacker approximant thereto) with the Laplacian of the electron density. Since these quantities, separately, are useful for elucidating shell structure, it is unsurprising that their combination would also be useful. Unfortunately, the introduction of the absolute value in (58) is a pragmatic choice, and does not have a strong theoretical motivation. Further studies of $\zeta_{\sigma}^{R}\left(\boldsymbol{r}_{i}\right)$ are clearly warranted.

It was observed that a transformation of the dependent variable (either $\xi_{\sigma}\left(\boldsymbol{r}_{i}\right)$ or $\zeta_{\sigma}\left(\boldsymbol{r}_{i}\right)$ ) is helpful for elucidating the shell structure in electron localization measures. The hyperbolic tangent form ((46) or, more generally, (48)), seems to be particularly useful in this context, and should be strongly considered as an alternative to the more commonly used transformations.

The final portion of this study investigated evaluting the electron localization function in the context of orbital-free density-functional theory. 
While this sometimes eliminates the time-consuming process of determining Kohn-Sham orbitals, unfortunately, the approximate kinetic energy density used here obscures (though does not eliminate) the shell structure in the electron localization function. It would be interesting to look at other, more sophisticated, models for the local kinetic energy density. ${ }^{37}$

\section{Acknowledgement}

The author would like to thank Prof. Andreas Savin and Prof. Robert Parr for numerous encouraging discussions on this (and other) topics. In addition, National Sciences \& Engineering Research Council of Canada and the Canada Research Chairs are thanked for funding.

\section{References}

1. Gillespie R J and Popelier P L A 2001 Chemical bonding and molecular geometry (New York: Oxford)

2. Daudel R 1953 C. R. Acad. Sci., Paris 237601

3. Daudel R, Brion H and Odiot S 1955 J. Chem. Phys. 232080

4. Aslangul R, Constanciel R, Daudel R and Kottis $\mathrm{P}$ 1972 Adv. Quantum Chem. 693

5. Daudel R 1968 The fundamentals of theoretical chemistry (Oxford: Pergamon)

6. Bader R F W and Stephens M E 1974 Chem. Phys. Lett. 26445

7. Daudel R, Bader R F W, Stephens M E and Borett D S 1974 Can. J. Chem. 521310

8. Daudel R, Bader R F W, Stephens M E and Borett D S 1974 Can. J. Chem. 523077

9. Bader R F W and Stephens M E 1975 J. Am. Chem. Soc. 977391

10. Chamorro E, Fuentealba P and Savin A 2003 J. Comput. Chem. 24496

11. Becke A D and Edgecombe K E 1990 J. Chem. Phys. 925397
12. Savin A, Nesper R, Wengert S and Fassler T F 1997 Angew. Chem. 361809

13. Savin A, Becke A D, Flad J, Nesper R, Preuss H and Vonschnering H G 1991 Angew. Chem. 30409

14. Schmider H L and Becke A D 2000 Theochem 52751

15. Schmider H L and Becke A D 2002 J. Chem. Phys. 1163184

16. Lieb E H 1981 Rev. Mod. Phys. 53603

17. Thomas L H 1927 Proc. Camb. Philos. Soc. 23542

18. Fermi E 1928 Z. Phys. 4873

19. Becke A D 1983 Int. J. Quantum Chem. 231915

20. Ghosh S K, Berkowitz M and Parr R G 1984 Proc. Natl. Acad. Sci. USA 818028

21. Berkowitz M 1986 Chem. Phys. Lett. 129486

22. Lee C and Parr R G 1987 Phys. Rev. A35 2377

23. The definition of the kinetic energy used here differs from the preceding by the Laplacian of the electron density. As shown by Ayers, Nagy, and Parr, omitting this factor can be justified.

24. Chattaraj P K, Chamorro E and Fuentealba P 1999 Chem. Phys. Lett. 314114

25. Ayers P W, Parr R G and Nagy A 2002 Int. J. Quantum Chem. 90309

26. Savin A 2004 J. Phys. Chem. Solids 652025

27. Kohout M 2004 Int. J. Quantum Chem. 97651

28. Kohn W and Sham L J 1965 Phys. Rev. 140 A1133A1138

29. Zhao Q, Morrison R C and Parr R G 1994 Phys. Rev. A50 2138

30. Colonna F and Savin A 1999 J. Chem. Phys. 110 2828

31. Wu Q and Yang W T 2003 J. Chem. Phys. 1182498

32. van Leeuwen R and Baerends E J 1994 Phys. Rev. A49 2421

33. Tsirelson V and Stash A 2002 Acta Crystallogr. $\mathbf{B 5 8}$ 780

34. Tsirelson V G 2002 Acta Crystallogr. B58 632

35. Tsirelson V and Stash A 2002 Chem. Phys. Lett. 351 142

36. Dreizler R M and Gross E K U 1990 Density functional theory: An approach to the quantum manybody problem (Berlin: Springer-Verlag)

37. Wang Y A and Carter E A 2000 Orbital-free kineticenergy density functional theory (ed.) S D Schwartz (Dordrecht: Kluwer) ch. 5, pp 117-184. 